\title{
Obsessive-Compulsive Disorder: Development of Demand- Controlled Deep Brain Stimulation with Methods from Stochastic Phase Resetting
}

\author{
Peter A Tass*,1,2, Joachim Klosterkötter ${ }^{3}$, Frank Schneider ${ }^{4}$, Doris Lenartz ${ }^{2}$, Anastasios Koulousakis ${ }^{2}$ and \\ Volker Sturm ${ }^{2}$ \\ 'Institute of Medicine, Research Center Jülich, Jülich, Germany; ${ }^{2}$ Department of Stereotactic and Functional Neurosurgery, University of Cologne, \\ Germany; ${ }^{3}$ Department of Psychiatry and Psychotherapy, University of Cologne, Germany; ${ }^{4}$ Department of Psychiatry and Psychotherapy, \\ Heinrich-Heine University, Düsseldorf, Germany
}

\begin{abstract}
Synchronization of neuronal firing is a hallmark of several neurological diseases. Recently, stimulation techniques have been developed which make it possible to desynchronize oscillatory neuronal activity in a mild and effective way, without suppressing the neurons' firing. As yet, these techniques are being used to establish demand-controlled deep brain stimulation (DBS) techniques for the therapy of movement disorders like severe Parkinson's disease or essential tremor. We here present a first conceptualization suggesting that the nucleus accumbens is a promising target for the standard, that is, permanent high-frequency, DBS in patients with severe and chronic obsessive-compulsive disorder (OCD). In addition, we explain how demand-controlled DBS techniques may be applied to the therapy of OCD in those cases that are refractory to behavioral therapies and pharmacological treatment.

Neuropsychopharmacology (2003) 28, S27-S34. doi: I 0. I 038/s..npp. 1300144
\end{abstract}

Keywords: stereotactic neurosurgery; deep brain stimulation; obsessive-compulsive disorder; phase resetting

\section{INTRODUCTION}

Obsessive-compulsive disorder (OCD) is a common chronic and disabling psychiatric disorder, which severely impairs personal, social, and professional life. Patients with OCD suffer from recurrent obsessions and uncontrolled compulsions like repetitive behavioral or mental acts occurring in response to an obsession (Goodman, 1999). Often OCD occurs together with depressive and other anxiety disorders. Positron emission tomography studies revealed hypermetabolism in the orbitofrontal cortex, caudate nucleus, and, sometimes, anterior cingulate cortex in patients with $\mathrm{OCD}$ (Saxena and Rauch, 2000). The main pathophysiological finding appears to be an abnormality in serotonin transmission (Micallef and Blin, 2001). Correspondingly, potent serotonin reuptake inhibitors are the pharmacotherapy of choice for OCD (Greist and Jefferson, 1998; Goodman, 1999; Micallef and Blin, 2001). If one psychopharmacological treatment turns out to be ineffective, it may be beneficial to switch to a different prescription and, additionally, to use proserotonergic and nonserotonergic medication (Greist and Jefferson, 1998; Goodman, 1999; Micallef and Blin, 2001). Besides drug therapy,

*Correspondence: Prof Dr PA Tass, Institute of Medicine, Research Center Jülich, 52425 Jülich, Germany, Fax: +49 246। 612820,

E-mail: P.Tass@fz-juelich.de

Received 01 September 2002; revised 03 November 2002; accepted

03 December 2002 behavioral therapy is the first choice of psychiatric intervention.

OCD is notorious for both its chronicity and its intractability. In severe, treatment-refractory OCD, the efficacy of neurosurgical procedures (cingulotomy, limbic leucotomy, and subcaudate tractotomy) is only modest (Jenike, 1998; Rauch et al, 2001). Some convincing results have been obtained with bilateral anterior capsulotomy (Meyerson, 1998; Lippitz et al, 1999).

Electrical deep brain stimulation (DBS) at high frequencies has a blocking effect on the stimulated target area and mimics the effect of tissue lesioning (Benabid et al, 1991; Blond et al, 1992) (Figure 1). DBS is reversible and has a much lower rate of side effects than lesioning with thermocoagulation (Schuurman et al, 2000). Thus, Nuttin et al (1999) used bilateral DBS of the anterior limb of the internal capsule instead of lesoning for the treatment of refractory severe OCD. A significant improvement of symptoms could be achieved in four patients, but unusually high stimulation amplitudes had to be used, which led to a high energy consumption. In order to improve the efficacy of DBS and to reduce the energy consumption, we propose two strategies:

1. Based on clinical observations as well as anatomical and pathophysiological considerations, the nucleus accumben is suggested as the primary target for DBS in OCD. 

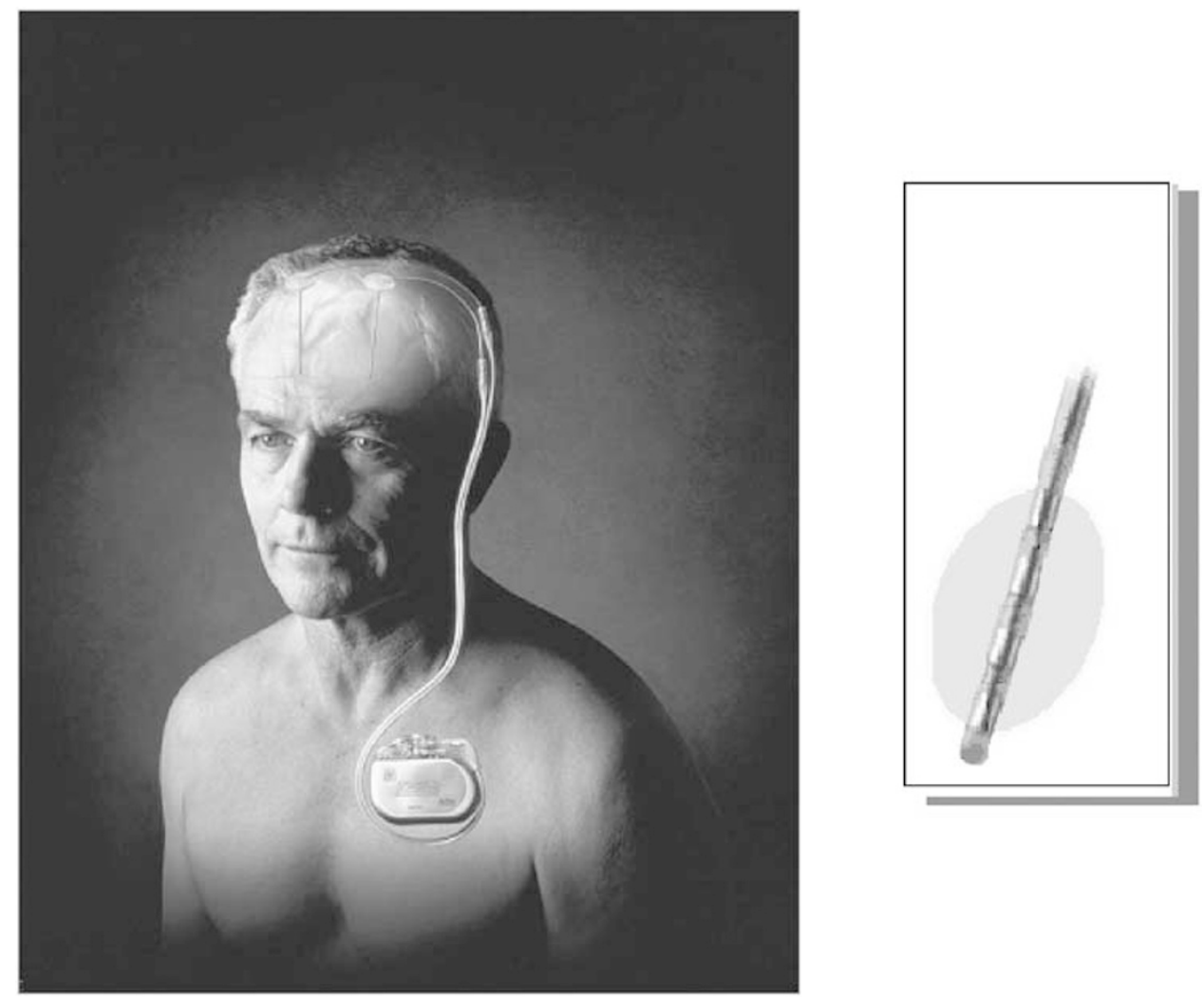

Figure I Left: Schematic drawing of two implanted electrodes connected to the stimulator, which is implanted subcutaneously below the clavicula. Right: DBS electrode with four leads, separated by $1.5 \mathrm{~mm}$.

2. Based on modern techniques from statistical physics, in particular, stochastic phase resetting (Tass, 1999), demand-controlled stimulation techniques have been developed, which enable an effective and mild desynchronization of synchronized oscillatory activity (Tass, 1999, 2001a, b, 2002a). As yet, these techniques have been used to design demand-controlled DBS techniques for the therapy of movement disorders like severe Parkinson's disease (PD) or essential tremor (Tass, 1999, 2001c, $2002 \mathrm{~b}$ ). We here explain how to apply these methods to the treatment of OCD.

\section{TARGET SELECTION AND PRELIMINARY RESULTS}

The extensive experience of the Karolinska group as well as the group of VS with observations in a limited number of patients with thermolesioning of the anterior limbs of the internal capsule seems to stress the importance of the ventro-caudal part of the anterior limb of the internal capsule as target for successful treatment of OCD (Meyerson, 1998). Similar findings have been reported by Rasmussen and Greenberg (personal communication) for gamma knife capsulotomy.

The ventral part of the anterior limb of the internal capsule is immediately adjacent to the nucleus accumbens, which has a central position between limbic structures, especially the amygdala, the basal ganglia, the DM thalamus, and the orbito-frontal region, into which it extends rostrally. All these structures seem to be important for the development of anxiety and OC symptoms. Thermocoagulations of the internal capsule frequently encroach into the nucleus accumbens. These considerations prompted one of us (VS) to modify the electrode track for DBS of the anterior limb of the internal capsule in a way that permitted stimulation of the ventral part of the anterior limb of the internal capsule as well as the nucleus accumbens and the rostro-caudal part of the bed nucleus of the stria terminalis, which receives strong inputs from central amygdala and projects to the rostrally and ventrally adjacent nucleus accumbens (Figure 2). The arrangement of the leads of the used electrodes (Medtronic, Minneapolis) allowed to reach these targets with one electrode and to stimulate selectively the respective structures (Figure 3).

Unilateral DBS of the right nucleus accumbens and the rostro-ventral edge of the bed nucleus of the stria terminalis with permanent pulse-train stimulation, that is square wave impulses of $90 \mu$ s impulse duration, $130 \mathrm{~Hz}$ and amplitudes between 2 and $4 \mathrm{~V}$, was applied to three patients with $\mathrm{OCD}$ (ICD-10 classification F42.2 'Mixed obsessional thoughts and acts') who were therapy resistant after long-term psychopharmacological and behavior therapy since many years. Therapy resistance was defined according to international standards. DBS clearly attenuated OCD symptoms in two of three patients with follow-up periods of 15-18 months. In the third patient no beneficial effect could be achieved. A recently performed MRI investigation revealed a displacement of the electrode in the caudo-ventral 


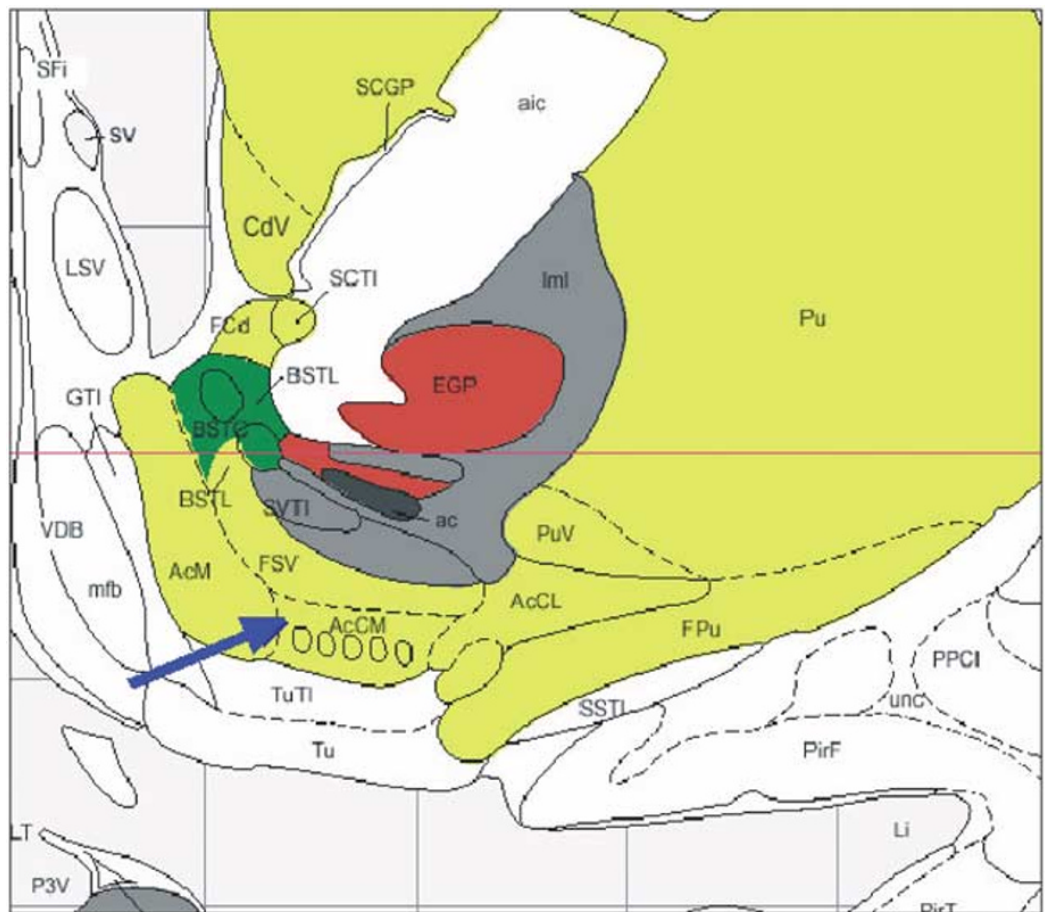

Figure 2 Frontal section through the target area, giving the topographic relations between internal capsule, nucleus accumbens, and bed nucleus of stria terminalis: Target point (head of arrow): $3 \mathrm{~mm}$ rostral CA, $7 \mathrm{~mm}$ right lateral of midline, 3-4 mm ventral of AC-PC line. Green: Rostral edge of bed nucleus of stria terminalis, white: caudal part of anterior limb of internal capsule (atlas of Mai et al, 1997, frontal section $2.7 \mathrm{~mm}$ rostral of CA).

Bed Nucleus

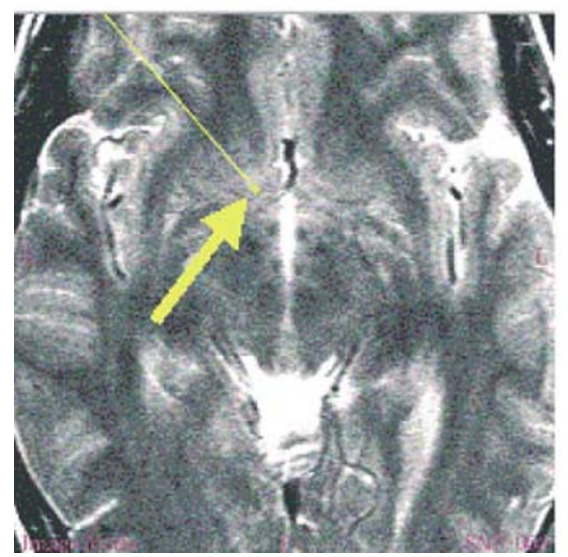

Nucl. Accumbens

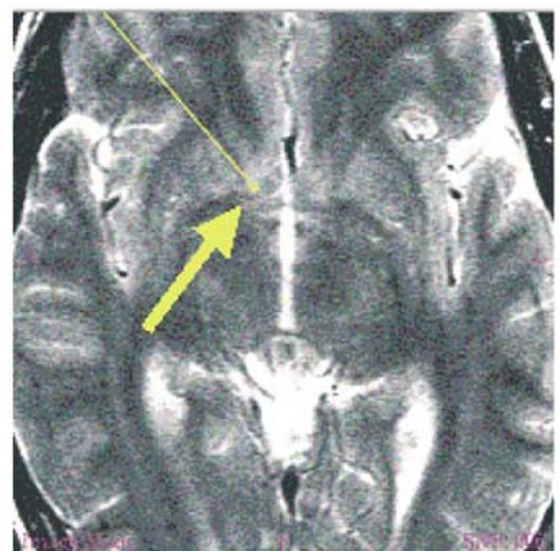

Int.

Capsule

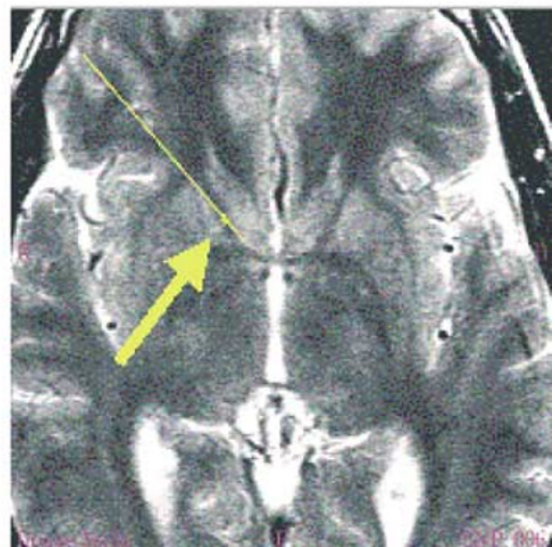

Figure 3 MR-based trajectory planning. The electrode tract penetrates the ventral part of the anterior part of the internal capsule (proximal electrode lead), nucleus accumbens (two middle leads), and reaches the rostro-caudal edge of the bed nucleus of the stria terminalis (distal lead).

direction and missing of the target area, which could explain the therapeutic failure. The results will be published in detail elsewhere.

\section{DEMAND-CONTROLLED DBS}

In several neurological diseases such as PD or essential tremor, brain function is severely impaired by pathological synchronization of neuronal firing. Parkinsonian resting tremor appears to be caused by a cluster of neurons located in the thalamus and the basal ganglia, which fire synchronously at a frequency similar to that of the tremor (Llinàs and Jahnsen, 1982; Pare et al, 1990; Lenz et al, 1994). In contrast, under physiological conditions these neurons fire incoherently (Nini et al, 1995). In patients with PD this cluster acts like a pacemaker and activates premotor cortex, supplementary motor area, and the motor cortex (Alberts et al, 1969; Lamarre et al, 1971; Bergman et al, 1994; Nini et al, 1995; Volkmann et al, 1996), where the latter synchronize their oscillatory activity (Tass et al, 1998). In patients with advanced PD or with essential tremor who do not respond to drug therapy any more, depth electrodes are 


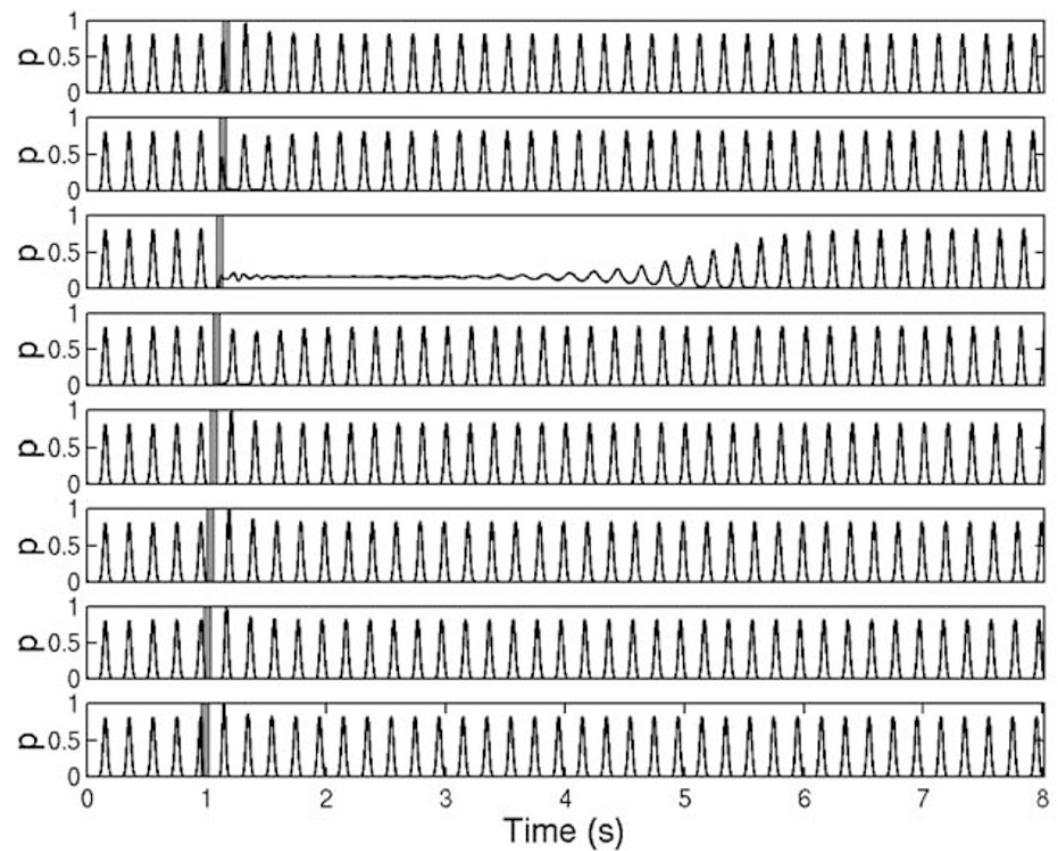

Figure 4 In a series of simulations, a population of neurons is stimulated with an identical single pulse (indicated by a bar), which is administered at different initial phases of the collective oscillation, where the initial phase is varied in equidistant steps within one cycle. The time course of the firing density is plotted, where the firing density is a quantity corresponding to the probability with which the population of neurons fires. The single pulse has to hit the population at a vulnerable phase in order to cause a transient desynchronization of the population. The resynchronization occurs due to the strongly synchronizing neuronal interactions within the population. Simulation taken from Tass (1999).

chronically implanted in target areas like the thalamic ventralis intermedius nucleus or the subthalamic nucleus (Benabid et al, 1991, 2000; Blond et al, 1992; Volkmann and Sturm, 1998). DBS at high frequencies suppresses the neuronal activity of the pacemaker-like cluster which, in turn, suppresses the peripheral tremor (Benabid et al, 1991; Blond et al, 1992). For this, a permanent high-frequency $(>100 \mathrm{~Hz})$ periodic pulse train is permanently administered via the depth electrode.

Clinical observations of subthalamic nucleus stimulation for the treatment of PD have indicated a potential for modulating emotional behavior and even a risk for developing depression or anxiety disorders in some patients. Chronic subthalamic nucleus stimulation, however, selectively enhanced affective processing and subjective well-being and seems to be antidepressive (Schneider et al, 2002). More positive self-reported mood and an enhanced mood induction effect as well as improvement in emotional memory during stimulation were observed. However, cognitive performance was not affected by the different conditions and treatments.

High-frequency DBS has been developed empirically, mainly based on observations during stereotactic surgery (Benabid et al, 1991; Blond et al, 1992). The mechanism by which DBS at high frequencies suppresses pathological rhythmic activity has not yet been clarified in detail. The permanent high-frequency stimulation basically mimics the effect of tissue lesioning by suppressing neuronal firing (Benabid et al, 1991, 2000; Blond et al, 1992; Wielepp et al, 2001). However, permanent high-frequency stimulation is an unphysiological input that may cause an adaptation of the stimulated neuronal networks. This may be one of the reasons why in a number of patients the stimulation amplitude has to be increased in the cause of the treatment in order to maintain a therapeutic effect. As a consequence of the increased stimulation strength, neighboring areas may be affected because of current spread, which leads to severe side effects like dysarthria, dysesthesia, and cerebellar ataxia.

Demand-controlled DBS techniques have been designed based on a stochastic phase-resetting theory (Tass, 1999) and are just being tested in patients during neurosurgery. The stochastic phase-resetting theory explains how stimuli affect synchronization and desynchronization processes in networks of oscillators, such as rhythmically firing or bursting neurons, in the presence of noise. The stochastic phase-resetting approach was developed with methods from statistical physics (Kuramoto, 1984) and synergetics (Haken, 1983). In this way, it became possible to extend the concept of phase resetting (Winfree, 1980; Glass and Mackey, 1988) to networks of oscillators subject to random forces. Taking into account the noise turned out to be essential for the design of stimulation techniques as well as data analysis tools necessary for their calibration (Tass, 1999). The two main features of the novel demandcontrolled DBS techniques are: (a) minimization of the current used for stimulation and (b) desynchronization of synchronized neuronal activity instead of simply suppressing neuronal firing. Put otherwise, the novel DBS techniques aim at a desynchronization on demand in order to maintain an uncorrelated firing in a neuronal population that tends to fire synchronously.

Desynchronization of a fully synchronized neuronal population can be achieved with a single electrical pulse of the right intensity and duration provided the pulse hits the population in a vulnerable phase range that corresponds 


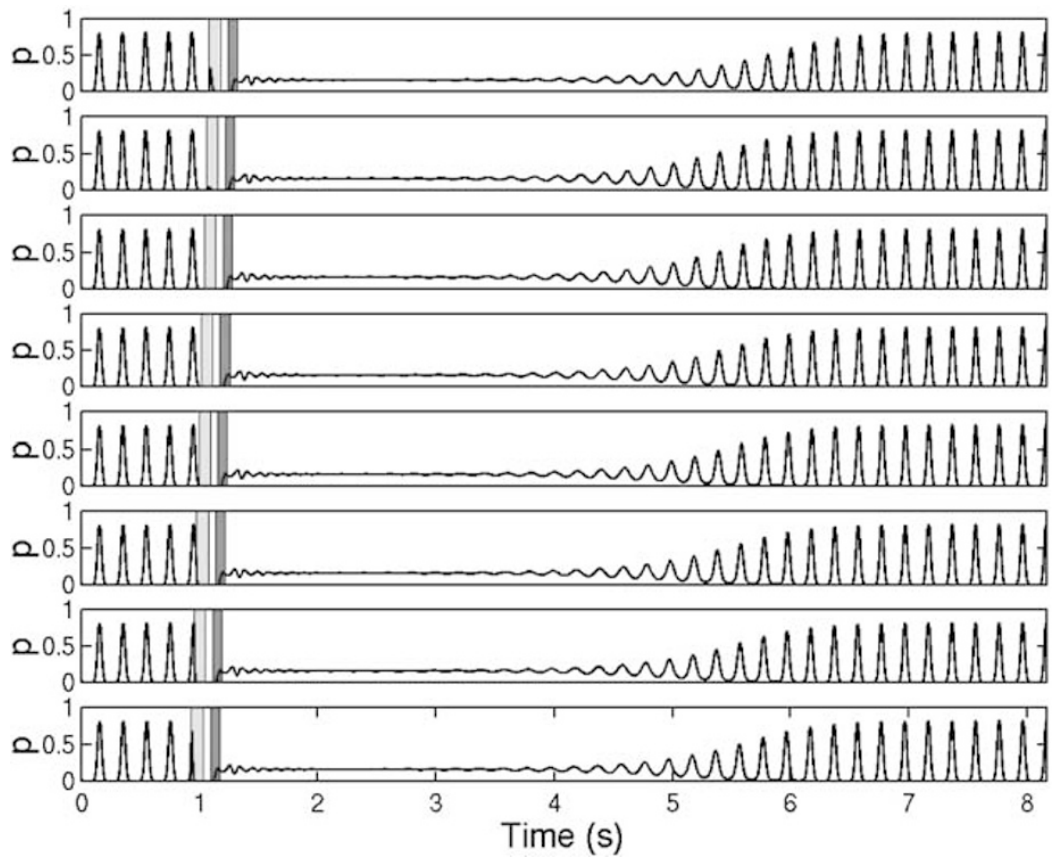

Figure 5 In a series of simulations the same population of neurons as in Figure 4 is stimulated with an identical double pulse (indicated by two bars) administered at different initial phases of the collective oscillation. The first, stronger pulse of the double pulse (left bar) resets the collective rhythm. The second, weaker pulse (right bar) follows with a constant delay and hits the population in a vulnerable state. The reset guarantees the phase-independent, effective desynchronization. Simulations taken from (Tass, 200 Ic).

to only a small fraction ( $5 \%$ or even less) of a period of the oscillation (Tass, 1999) (Figure 4). Of course, this is tricky to be realized under noisy experimental conditions typically encountered in the brain. Furthermore, the right stimulation parameters (ie intensity, duration, and vulnerable phase) necessary for an effective desynchronization also depend on the extent of the cluster's synchronization. To desynchronize a weakly synchronized cluster, a weaker pulse has to be used. For medical applications, it is preferable to use a stimulus that always causes a desynchronization, independent of the cluster's dynamical state. For this reason, composite stimulation techniques have been developed (Tass, 2001a-c, 2002a, b). A composite stimulus consists of two qualitatively different stimuli: The first stimulus controls the cluster's dynamics by resetting (ie restarting) it. After the first stimulus, the cluster restarts in a stereotyped manner. The second stimulus is administered with a fixed delay after the first stimulus. Owing to the stereotyped reset, the second pulse hits the cluster in a vulnerable state, in this way causing a desynchronization (Figure 5).

The first stimulus of a composite stimulus is either a strong single pulse (Tass, 2001a,c) or a high-frequency pulse train (with a frequency that exceeds the cluster's natural frequency by a factor of 20 or more) (Tass, 2001b) (Figure 6a,b). For example, in the case of PD, the resetting high-frequency pulse train would be a short epoch of highfrequency $(>100 \mathrm{~Hz})$ stimulation. The minimal length of this epoch necessary to achieve a reset has to be calibrated (Tass, 2001b, 2002b). The strong first stimulus causes a hard reset (ie abrupt reset) during which the collective oscillation runs through less than one cycle. A hard reset requires that the stimulus strongly affects the stimulated neuronal population without causing any damage to the tissue. Alternatively, in patients who do not tolerate strong stimuli, a different strategy can be pursued by using a first stimulus that causes a soft reset (ie slow reset, Figure 6c) (Tass, 2002a, b). A soft reset is realized with a low-frequency pulse train, where pulses are periodically administered at a rate similar to the natural frequency of the neuronal population. During a soft reset the collective oscillation is not quickly stopped. Rather by entraining the cluster to the lowfrequency stimulation, the influence of the initial dynamic state at the beginning of the stimulation fades away while the collective oscillation runs through several cycles. This is done to control the cluster's dynamics, so that the second stimulus, a single pulse that follows after a constant time delay, desynchronizes the cluster by hitting it in a vulnerable state. A soft reset has already been studied by Hassler et al (1960). They observed that a periodic deep brain electrical stimulation of the pallidum at rates similar to the peripheral tremor frequency caused an entrainment of the peripheral tremor. However, a desynchronizing stimulation has not yet been applied.

Since a combined stimulus desynchronizes a cluster independent of the initial dynamical state at which it is administered, it can be used to block the cluster's resynchronization. To this end a combined stimulus has to be administered repeatedly, whenever the cluster becomes synchronized again (Figure 6). The goal of this stimulation mode is to avoid a pathological synchronization and to maintain an uncorrelated firing. A detailed comparison of the different demand-controlled stimulation techniques based on stochastic phase resetting together with 

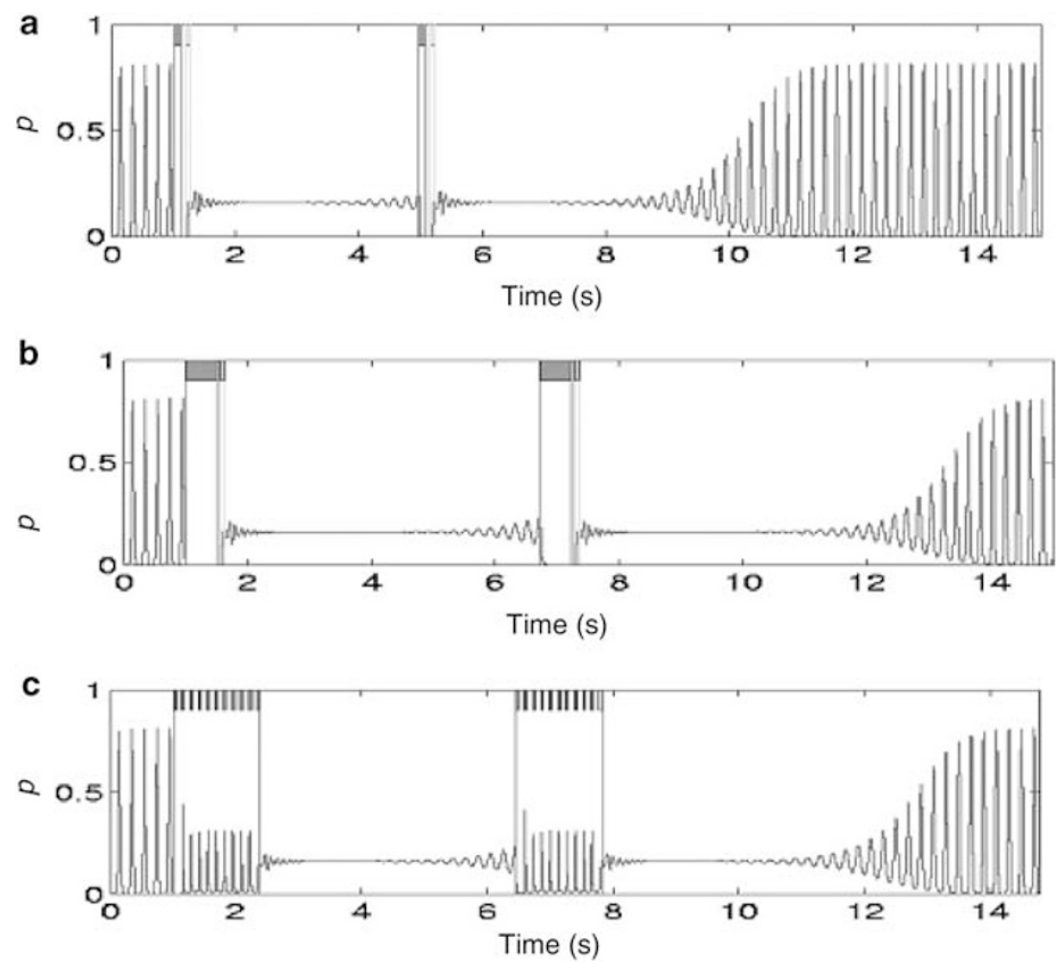

Figure 6 Resynchronization is blocked by an intermittent, demand-controlled stimulation. To this end the same stimulus is administered whenever the extent of synchronization (ie the amplitude of the collective firing) exceeds a critical value. Effectively desynchronizing stimuli consist of two qualitatively different stimuli: a first, stronger stimulus followed by a second, weaker stimulus after a constant delay. The first stimulus resets the collective oscillation and may either be a strong pulse $(a)$, a high-frequency $(>100 \mathrm{~Hz})$ pulse train $(b)$ or a low-frequency pulse train with a pulse rate in the range of the population's frequency (c). A strong pulse as well as a high-frequency pulse train cause a hard reset, where the collective oscillation is abruptly stopped (a,b). In contrast, during a low-frequency reset $(c)$ the collective oscillation gets entrained, ie the population adapts the rhythm of the periodic sequence of pulses. The second stimulus is a desynchronizing pulse which acts as shown in Figure 4. In (a) and (b) begin and end of the stimuli are indicated by vertical lines connected by a short bar (at the top). In (c) begin and end of a composite stimulus are indicated by vertical lines; each single pulse corresponds to a short bar (at the top). Simulations taken from (Tass, 200 lc, 2002b).

their calibration procedures was presented elsewhere (Tass, 2002b).

\section{DISCUSSION}

The significant improvement of formerly treatment refractory severe OC- and anxiety symptoms obtained with unilateral high-frequency stimulation of the right nucleus accumbens and the rostro-caudal part of the bed nucleus of the stria terminalis seem to indicate a major role of the nucleus accumbens as a central relay structure between amygdala, the basal ganglia, the DM thalamus, and the orbito-frontal cortex. The amygdaloid complex is well known to be decisive for negative emotions like sadness, anxiety, and fear reactions, and characteristic dysfunctions in psychiatric patients have been described (Schneider et al, 1996, 1997; Davis, 2000). The main mentioned projection areas of the nucleus accumbens, mainly the basal ganglia and the orbitofrontal cortex, are thought to be morphological substrates of OCD (Saxena and Rauch, 2000).

The importance of the nucleus accumbens is further stressed by the reports of beneficial effects of subcaudate tractotomy on OCD, anxiety disorders, and depression (Knight, 1969), a bilateral procedure that would interrupt parts of the nucleus accumbens. The finding that, different from the classical bilateral anterior capsulotomy and DBS of the internal capsule in OCD (Meyerson, 1998; Nuttin et al, 1999), right-sided DBS of the nucleus accumbens was sufficient to achieve significant clinical improvement is supported by Lippitz et al (1999) who found right-sided thermo-lesions in the middle part of the anterior limb of the internal capsule to be decisive for a good therapeutic outcome.

The fact that much lower current amplitudes had been sufficient in our two successfully treated patients than in the series on bilateral stimulation of the anterior limb reported by Nuttin et al (1999) as well as the negative results in our third patient, in whom the target area was missed, points to the importance of our chosen target structures in the genesis of OCD and anxiety disorders.

Based on the theoretical studies reviewed in the section 'Demand-controlled deep brain stimulation', we suggest demand-controlled DBS as a possible therapy of severe OCD-patients who are refractory to behavioral therapies and pharmacological treatment and, thus, severely disabled. Demand-controlled DBS can be applied on condition that (a) pathologically strong synchronization within a neuronal population is relevant for the emergence of OC symptoms and (b) it is possible to register the neuronal activity of this particular area, for example, with a depth electrode or an epicortical electrode. This area does not need to be identical 
with the target area where the electrode is located. It might also be an area that is closely connected to the target area. For example, a depth electrode used for stimulation might be located in the nucleus accumbens, whereas an epicortical electrode used for measuring the feedback signal might be placed over an area of the orbito-frontal cortex. To test whether conditions (a) and (b) are fulfilled, depth recordings as well as EEG measurements have to be performed, for example, during neurosurgery in patients with OCD.

If pathological synchronization is relevant in OCD, demand-controlled DBS should be applied as sketched in Figure 6. The electrical activity measured with a depth electrode or an epicortical electrode serves as feedback signal. Whenever the amplitude of the feedback signal in the relevant frequency band exceeds a critical value, a desynchronizing stimulus is administered to avoid pathological synchronization and to maintain an uncorrelated firing. This might enable to perform a milder DBS therapy in patients suffering from OCD.

\section{REFERENCES}

Alberts WW, Wright EJ, Feinstein B (1969). Cortical potentials and parkinsonian tremor. Nature 221: 670-672.

Benabid A-L, Koudsieé A, Benazzouz A, Fraix V, Ashraf A, Le Bas JF et al (2000). Subthalamic stimulation for Parkinson's disease. Arch Med Res 31: 282-289.

Benabid AL, Pollak P, Gervason C, Hoffmann D, Gao DM, Hommel $M$ et al (1991). Long-term suppression of tremor by chronic stimulation of the ventral intermediate thalamic nucleus. The Lancet 337: 403-406.

Bergman H, Wichmann T, Karmon B, DeLong MR (1994). The primate subthalamic nucleus. II: Neuronal activity in the MPTP model of parkinsonism. J Neurophysiol 72: 507-520.

Blond S, Caparros-Lefebvre D, Parker F, Assaker R, Petit H, Guieu $\mathrm{J}$-D et al (1992). Control of tremor and involuntary movement disorders by chronic stereotactic stimulation of the ventral intermediate thalamic nucleus. J Neurosurg 77: 62-68.

Davis M (2000). The role of the amygdala in conditioned and unconditioned fear and anxiety. In: Aggleton JP (ed). The amygdala, a functional analysis. Oxford University Press: Oxford. pp 214-287.

Glass L, Mackey MC (1988). From clocks to chaos. The rhythms of life. Princeton University Press: Princeton.

Goodman WK (1999). Obsessive-compulsive disorder: diagnosis and treatment. J Clin Psychiatry 60(Suppl 18): 27-32.

Greist JH, Jefferson JW (1998). Pharmacotherapy for obsessivecompulsive disorder. Br J Psychiatry (Suppl): 64-70.

Haken H (1983). Advanced Synergetics. Springer: Berlin.

Hassler R, Riechert T, Mundinger F, Umbach W, Ganglberger JA (1960). Physiological observations in stereotaxic operations in extrapyramidal motor disturbances. Brain 83: 337-350.

Jenike MA (1998). Neurosurgical treatment of obsessive-compulsive disorder. Br J Psychiatry (Suppl): 79-90.

Knight GC (1969). Bi-frontal stereotactic tractotomy: an atraumatic operation of value in the treatment of intractable psychoneurosis. Br J Psychiatry 115: 257-266.

Kuramoto Y (1984). Chemical Oscillations, Waves, and Turbulence. Springer: Berlin.

Lamarre Y, DeMontigny C, Dumont M, Weiss M (1971). Harmaline-induced rhythmic activity of cerebellar and lower brain stem neurons. Brain Res 32: 246-250.

Lenz FA, Kwan HC, Martin RL, Tasker RR, Dostrovsk JO, Lenz YE (1994). Single unit analysis of the human ventral thalamic nuclear group. Tremor-related activity in functionally identified cells. Brain 117: 531-543.
Lippitz BE, Mindus P, Meyerson BA, Kihlstrom L, Lindquist C (1999). Lesion topography and outcome after thermocapsulotomy or gamma knife capsulotomy for obsessive-compulsive disorder: relevance of the right hemisphere. Neurosurgery 44: 452-458.

Llinàs R, Jahnsen $H$ (1982). Electrophysiology of mammalian thalamic neurons in vitro. Nature 297: 406-408.

Mai JK, Assheuer J, Paxinos G (1997). Atlas of the human brain. Academic Press: San Diego.

Meyerson BA (1998). Neurosurgical treatment of mental disorders: introduction and indications. In: Gildenberg PL, Tasker RR (eds). Textbook of stereotactic and functional neurosurgery. Mc Graw Hill. pp 1953-1963.

Micallef J, Blin O (2001). Neurobiology and clinical pharmacology of obsessive-compulsive disorder. Clin Neuropharmacol 24: 191207.

Nini A, Feingold A, Slovin H, Bergman H (1995). Neurons in the globus pallidus do not show correlated activity in the normal monkey, but phase-locked oscillations appear in the MPTP model of parkinsonism. J Neurophysiol 74: 1800-1805.

Nuttin B, Cosyns P, Demeulemeester H, Gybels J, Meyerson B (1999). Electrical stimulation in anterior limbs of internal capsules in patients with obsessive-compulsive disorder. The Lancet 354: 1526.

Pare D, Curro'Dossi R, Steriade M (1990). Neuronal basis of the parkinsonian resting tremor: a hypothesis and its implications for treatment. Neuroscience 35: 217-226.

Rauch SL, Dougherty DD, Cosgrove GR, Cassem EH, Alpert NM, Price BH et al (2001). Cerebral metabolic correlates as potential predictors of response to anterior cingulotomy for obsessive compulsive disorder. Biol Psychiarty 50: 659-667.

Saxena S, Rauch SL (2000). Functional neuroimaging and the neuroanatomy of obsessive-compulsive disorder. Psychiatr Clin North Am 23: 563-586.

Schneider F, Grodd W, Weiss U, Klose U, Mayer KR, Nägele T et al (1997). Functional MRI reveals left amygdala activation during emotion. Psychiatry Res: Neuroimaging 76: 75-82.

Schneider F, Gur RE, Alavi A, Seligman MEP, Harper Mozley L, Smith RJ et al (1996). Cerebral blood flow changes in limbic regions induced by unsolvable anagram tasks. Am J Psychiatry 153: 206-212.

Schneider F, Habel U, Volkmann J, Regel S, Kornischka J, Sturm V et al (2002). Deep brain stimulation of the subthalamic nucleus enhances emotional processing in Parkinson's disease. submitted.

Schuurman PR, Bosch DA, Bossuyt PM, Bonsel GJ, van Someren EJ, de Bie RM et al (2000). A comparison of continuous thalamic stimulation and thalamotomy for suppression of severe tremor. $N$ Engl J Med 342: 461-468.

Tass PA (1999). Phase Resetting in Medicine and Biology-Stochastic Modelling and Data Analysis. Springer: Berlin.

Tass PA (2001a). Effective desynchronization by means of doublepulse phase resetting. Europhys Lett 53: 15-21.

Tass PA (2001b). Effective desynchronization with a resetting pulse-train followed by a single pulse. Europhys Lett 55: 171-177.

Tass PA (2001c). Desynchronizing double-pulse phase resetting and application to deep brain stimulation. Biol Cybern 85: 343354.

Tass PA (2002a). Effective desynchronization with a stimulation technique based on soft phase resetting. Europhys Lett 57: 164170.

Tass PA (2002b). Desynchronization of brain rhythms with soft phase-resetting techniques. Biol Cybern 87: 102-115.

Tass PA, Rosenblum MG, Weule J, Kurths J, Pikovsky A, Volkmann J et al (1998). Detection of n:m phase locking from noisy data: application to magnetoencephalography. Phys Rev Lett 81: 3291-3294. 
Volkmann J, Joliot M, Mogilner A, Ioannides AA, Lado F, Fazzini E et al (1996). Central motor loop oscillations in parkinsonian resting tremor revealed by magnetoencephalography. Neurology 46: 1359-1370.

Volkmann J, Sturm V (1998). Indication and results of stereotactic surgery for advanced Parkinson's disease. Crit Rev Neurosurg 8: 209-216.

Wielepp JP, Burgunder JM, Pohle T, Ritter EP, Kinser JA, Krauss JK (2001). Deactivation of thalamocortical activity is responsible for suppression of parkinsonian tremor by thalamic stimulation: a 99mTc-ECD SPECT study. Clin Neurol Neurosurg 103: 228231.

Winfree AT (1980). The Geometry of Biological Time. Springer: Berlin. 